\title{
Produção Científica sobre o Teste de Cloze
}

\author{
Adriana Cristina Boulhoça Suehiro \\ Universidade Federa do Recôncavo da Bahia - BA
}

\begin{abstract}
Resumo
O presente estudo investigou a produção científica sobre o Cloze publicada entre os anos de 2002 e 2012. Foram focalizados 14 periódicos, nos quais 32 artigos foram analisados com base em alguns critérios estabelecidos por Witter e outros considerados relevantes pela autora. Os dados evidenciaram um aumento das publicações a partir de 2007, sendo os anos de 2007 e 2008 os mais férteis. Observou-se que o relato de pesquisa foi a forma mais utilizada e que o Sudeste foi a região que mais publicou nesse período. Houve ainda a predominância de autorias múltiplas e femininas e de pesquisas que associaram outros testes ou escalas ao Cloze, portanto, houve relações com outros construtos. Os resultados apontaram também a predominância de pesquisas no contexto educacional e com universitários, baseadas no emprego da técnica tradicional, com a supressão de todo quinto vocábulo do texto e correção literal.
\end{abstract}

Palavras-chave: Bibliometria, compreensão de leitura, remediação de leitura.

\section{Scientific Production on Cloze Test}

\begin{abstract}
In this study we investigate the scientific production on Cloze test, between the years 2002 and 2012. Fourteen journals were used, from which 32 articles were analyzed on the basis of certain criteria set by Witter and others considered relevant by the author. The data evidenced an increase in publications since 2007. The years 2007 and 2008 were the most productive. The research report was the most used form and the southeastern region the one which most published in that period. There was still the predominance of multiple and feminine authorship and studies that employed other tests to the Cloze, and therefore revealed a relationships with other constructs. The results also pointed to the predominance of research in educational context with university student, based on the use of traditional technique, with the deletion of every fifth word of the text and literal correction.
\end{abstract}

Keywords: Bibliometric, reading comprehension, remedial reading.

\section{Producción científica sobre el Test de Cloze}

\section{Resumen}

El presente estudio investigó la producción científica sobre el Cloze entre los años 2002 y 2012. Se enfocaron 14 periódicos de los cuales se analizaron 32 artículos con base en algunos criterios establecidos por Witter y otros considerados relevantes por la autora. Los datos mostraron un aumento en las publicaciones desde 2007, siendo los años de 2007 y 2008 los más proficuos. Se observó que el relato de investigación fue la forma más utilizada y que el Sudoeste fue la región que más publicó en este período. Asimismo, hubo predominancia de autoría múltiple y femenina, así como de investigaciones que asociaron otros tests o escalas al Cloze y, por lo tanto, hubo relaciones con otros constructos. Los resultados indicaron también para la predominancia de investigaciones en contexto educacional y con universitarios, basadas en el uso de la técnica tradicional con supresión de todos los quintos vocablos del texto y corrección literal.

Palabras clave: Bibliometria, compresión de lectura, remediación de la lectura. 


\section{Introdução}

A técnica de Cloze foi desenvolvida por Taylor, em 1953, e consiste na organização de um texto do qual se omitem alguns vocábulos e no qual se pede ao leitor que, após sua leitura integral, preencha as lacunas com as palavras que melhor completem o sentido do texto. A alta aceitação da técnica tem sido demonstrada pelas inúmeras publicações discorrendo sobre o leque de possibilidades de utilização desse recurso (Bensoussan, 1990; Kletzien, 1991; Kopke Filho, 2001; Pinto, Alvarenga, \& Kock, 1997; Santos, 1990; Vicentelli, 1999).

Considerada como um procedimento simples e flexível, que não coloca intermediários entre o leitor e o texto, porque o texto é o próprio teste, a preparação dessa técnica segue regras que variam em função do objetivo para o qual o texto será utilizado. Os parâmetros mais frequentes na omissão sistemática das palavras consistem na retirada de todo quinto, sétimo ou décimo vocábulo, na supressão de uma categoria gramatical (adjetivos, substantivos, verbos ou outras), ou ainda na eliminação aleatória de $20 \%$ dos vocábulos do texto (Bormuth, 1968; Braga, 1981; Giordano, 1985; Grant, 1979; Helfeldt \& Henk, 1985; Hines \& Warren, 1978; Porter, 1979; Riley, 1986; Santos, 2004; Zucoloto, 2001).

Além da variação na utilização dos parâmetros de omissão das palavras, há também diferenças relativas à sua apresentação. De acordo com Santos (2004), o texto de Cloze é geralmente apresentado por escrito, sendo a palavra omitida substituída por um traço de tamanho sempre igual, tal como propõe Taylor (1953), ou proporcional ao tamanho do vocábulo omitido, como sugerido por Bormuth (1968). Isso porque, de acordo com Bormuth (1968), os resultados obtidos com a utilização do traço proporcional apresentam um índice mais alto de correlação com outras medidas de compreensão em leitura.

Quanto à estruturação do texto, Bitar (1989) salienta que várias são as diversificações sofridas em relação à forma tradicional proposta por Taylor. Entre as variações surgiram o Cloze lexical, o Cloze gramatical, o Cloze de múltipla escolha e o Cloze Cumulativo. No Cloze lexical, somente os itens lexicais do texto são omitidos, enquanto no Cloze gramatical se omitem todos os itens relacionais (palavras que não têm significado próprio e servem para conectar outras palavras estruturando o texto sintaticamente), ou seja, preposições, conjunções, entre outros. No que se refere ao Cloze de múltipla escolha, são oferecidas múltiplas alternativas para o preenchimento da lacuna; finalmente, no Cloze cumulativo há a omissão sistemática de uma única palavra, a qual é substituída por uma palavra sem sentido. Destarte, nesta variação, a tarefa do leitor é identificar a palavra sem sentido cada vez que ela apareça no texto.

Em seu estudo, Bitar (1989) comparou instrumentos de diagnóstico em leitura quanto ao tipo de teste e ao critério de correção utilizado entre eles e verificou o comportamento dos leitores ao solucionar cada um deles. Sessenta crianças de segunda, terceira e quarta séries do conhecido na época como primeiro grau responderam a quatro testes de compreensão em leitura, a saber, o teste de atividades tradicionais, o teste de Cloze convencional, o teste de Cloze gramatical e, por fim, o teste de Cloze lexical. Os dados encontrados evidenciaram que o teste de Cloze convencional e o teste de atividades tradicionais se correlacionaram quando os textos empregados exigiam o uso da capacidade interpretativa do leitor, além da capacidade de compreensão. Os resultados sugeriram, ainda, que o Cloze convencional indicou com maior consistência o grau de maturidade do leitor. Não obstante, a autora ressalta a necessidade de se aprofundar a investigação para averiguar sua eficiência em relação ao teste de Cloze lexical. Já o teste de Cloze gramatical, segundo as análises, favoreceu o mascaramento das reais habilidades de leitura. No que se refere ao comportamento dos leitores na resolução das tarefas, Bitar (1989) observou uma consistência para cada nível de desempenho considerado.

No que se refere à estruturação do texto, além dos parâmetros de omissão das palavras, é preciso considerar também a questão de sua dificuldade e dos itens ou palavras omitidas. Quanto à dificuldade, verifica-se que o assunto abordado no texto também é um aspecto que deve ser considerado, tendo em vista que foram encontradas diferenças de desempenho com relação a essa variável; logo, sob essa perspectiva, a familiaridade com o assunto aumenta a probabilidade de acerto das respostas dadas, o que introduz uma variável interveniente na comparação dos desempenhos obtidos pelos indivíduos durante a realização da técnica (Cohen, 1975; Oliveira, Boruchovitch, \& Santos, 2009; Page, 1975).

Ao lado disso, Santos (1991) verificou que os erros cometidos pelos leitores ocorriam com maior frequência em palavras de determinadas classes gramaticais. Das categorias mais difíceis às mais fáceis, observou adjetivos, advérbios, substantivos, verbos, pronomes, contrações, conjunções, preposições e artigos. A autora concluiu que as categorias com forte carga semântica (adjetivos, substantivos, verbos e advérbios) tenderam a se mostrar mais difíceis do que as categorias de relatores, como artigos e pronomes, por exemplo.

A técnica de Cloze, como meio de avaliação da compreensão da leitura, apresenta também outras vantagens. Uma delas é o fato de o leitor contar com o contexto como único apoio e não se correr o risco de que ele use palavras-chaves da pergunta para adivinhar a resposta a ser dada, evitando, dessa forma, a utilização de perguntas cuja compreensão poderia ser mais difícil do que a própria leitura (Bensoussan, 1990; Garrido, 1988; Pellegrini, 1996; entre outros). Outra vantagem está relacionada à utilização da técnica como um meio de desenvolvimento da compreensão da leitura, ao permitir ao aluno adivinhar as palavras que são omitidas no texto, graças ao seu domínio das estruturas semântico-sintáticas da linguagem e a seus conhecimentos 
anteriores acerca do conteúdo do texto (Bitar, 1989; Giordano, 1985; Santos, 1990, 1991).

Tendo em vista as vantagens da técnica de Cloze, muitas pesquisas têm sido realizadas. Entre elas estão as ligadas à validação do instrumento, bem como as relacionadas à compreensão e à inteligibilidade (Carelli, 1992; Garrido, 1979; Haugh; 1975; Oliveira, Boruchovitch, \& Santos, 2007; Rankin; 1970; Zucoloto \& Sisto, 2002). Também há outras referentes à intervenção e ao processo de remediação da leitura (Braga, 1986; Coelho \& Correa, 2010; Hussein, 2008; Sampaio \& Santos, 2002; Santos, 1994; Santos \& Oliveira, 2010), entre muitos outros temas.

A maioria dos estudos publicados tem sido realizada com universitários (Arouca, 1997; Bensoussan, 1990; Bitar, 1989; Capovilla \& Santos, 2001; Oliveira \& Santos, 2008; Oliveira, Suehiro \& Santos, 2004; Rinaudo \& Olmos, 1996; Santos, 1991; Santos, 1997; Santos, Suehiro \& Oliveira, 2004; Santos, Vendramini, Suehiro \& Santos, 2006; Vicentelli, 1999; Witter, 1996). Em menor número encontram-se alguns que têm focalizado a população adolescente e infantil (Bampi, 2000; Bitar, 1989; Braga, 1981; Carelli, 1992; Castelo Branco, 1992; Castro, 1981; Joly, 1999; Joly \& Lomônaco, 2003; Neves, 1997; Oliveira, Boruchovitch, \& Santos, 2007; Santos, 2004, 2005; Santos \& Oliveira, 2010; Suehiro \& Santos, 2009; Zucoloto, 2001; Zucoloto \& Sisto, 2002).

Dentre os estudos realizados com a população adolescente e infantil, a maioria tem se dedicado ao processo de remediação em preferência a investigações que verifiquem a compreensão em leitura e atestem a validade do instrumento para a avaliação dessa habilidade, possibilitando o uso adequado da técnica e das informações que dela provêm e garantindo-Ihe maior legitimidade. De modo geral, esses estudos têm evidenciado que a técnica de Cloze é um instrumento que possibilita, de maneira eficiente, o treinamento em habilidades que favorecem a compreensão em leitura (Bampi, 2000; Braga, 1981; Carelli, 1992; Castro, 1981; Joly, 1999; Joly \& Lomônaco, 2003; Santos, 2004).

Apesar da importância e da facilidade de emprego dessa técnica e das inúmeras pesquisas realizadas com seu emprego, foi localizado apenas um estudo que se deteve à análise da produção científica voltada ao Cloze. Aliás, embora haja consenso quanto à relevância da análise da produção científica, poucos estudos específicos sobre a produção da psicologia têm sido realizados e registrados na literatura brasileira. As pesquisas que têm se debruçado sobre a produção científica nessa área do conhecimento têm revelado, por um lado, que a Região Sudeste tem se firmado como o grande berço da produção do país, e, por outro, que os manuscritos divulgados - em sua maioria, relatos de pesquisa - são escritos predominantemente por mulheres e em sistema de coautoria (Aguiar Netto, 1988; Cunha, Suehiro, Oliveira, Pacanaro, \& Santos, 2009; Oliveira e cols., 2007; Oliveira, Cantalice, Joly, \& Santos, 2006; Souza Filho, Belo, \& Gouveia, 2006; Suehiro, Cunha, Oliveira \& Pacanaro, 2007; Suehiro, Cunha, \& Santos, 2007; Suehiro, Gaino, \& Meireles, 2008, no prelo; Suehiro, Rueda, Oliveira, \& Paca- naro, 2009; Suehiro \& Rueda, 2009; Yamamoto, Souza, \& Yamamoto, 1999).

O único estudo localizado, em que o Cloze foi foco de análise e que faz algumas considerações quanto às publicações com o mesmo foi o de Cunha (2009). A autora recuperou 52 trabalhos que utilizaram a técnica de Cloze no período de 1976 a 2007. O ano com maior concentração de pesquisas foi o de 2000. Dos trabalhos produzidos com a técnica, $50 \%$ foram realizados a partir de então, e o tipo de estruturação do texto ou modalidade mais utilizada foi a tradicional.

A autora ressalta, ainda, que dos 52 trabalhos analisados, 47 foram realizados no contexto educacional e, em sua maioria, com estudantes universitários. No que se refere à relação do Cloze com outros testes, questionários, escalas e provas, os resultados evidenciaram que nas pesquisas em que houve correlação com a escrita utilizou-se a produção de textos escritos com tema livre, tema estabelecido e à vista de uma gravura e a Escala de Avaliação de Dificuldades na Aprendizagem da Escrita (ADAPE). Testes de múltipla escolha, de desempenho verbal, de descrição, questões sobre as dificuldades percebidas e sobre as estratégias utilizadas para a sua superação foram empregados quando se avaliou a interpretação de textos.

O teste de criatividade de Torrance, o de criticidade e o de criatividade também aparecem entre os instrumentos associados ao Cloze, assim como a Escala de Estratégias de Leitura, de estratégias de aprendizagem e de avaliação de estilos cognitivos. Houve ainda o uso de questionários como o de caracterização do comportamento de ler, de caracterização dos tipos mais frequentes de avaliação utilizados no Ensino Superior e de questões objetivas de matemática, assim como provas de raciocínio indutivo, lógico-dedutivo e de conhecimentos gerais. Outros tipos de resultados também foram correlacionados com o Cloze, tais como os resultados do vestibular, as médias acadêmicas, o Desenho da Figura Humana (de Koppitz), o método qualitativo de análise de Witter e o desempenho em jogos, assim como exercícios de relaxamento.

De acordo com Cunha (2009), seus resultados corroboram a versatilidade e utilidade da técnica, pois o teste assumiu diversos formatos nas pesquisas recuperadas. Do mesmo modo, tais achados ratificaram a utilidade do teste de Cloze tanto para o diagnóstico da compreensão em leitura, utilizado em $71,5 \%$ das pesquisas, quanto para o desenvolvimento de programas de intervenção e remediação, que representou $21,5 \%$ dos trabalhos analisados.

Diante do exposto, considera-se que, independentemente do meio de divulgação, pesquisas sistemáticas sobre a produção científica de um determinado tema ou assunto são imprescindíveis para revelar o "estado da arte" do conhecimento em um campo de estudo e promovem crescimento, uma vez que sua divulgação facilita o acesso ao conhecimento em diversas áreas. Ao lado disso, possibilitam o levantamento de possíveis diretrizes para novos temas de pesquisas e de distribuição de fomento (Freitas, 1998; 
Witter, 1996, 1999, 2006). Considerando esses aspectos, o presente estudo objetivou analisar a produção científica sobre o teste de Cloze, entre 2002 e 2012, em periódicos científicos de psicologia.

\section{Método}

O estudo foi realizado em três etapas. A primeira focalizou todos os periódicos disponibilizados no Scientific Electronic Library Online (SciELO) e nos Periódicos Eletrônicos em Psicologia (PEPSIC); a segunda, apenas aqueles nos quais foram encontrados artigos que focalizavam o Teste de Cloze na época da coleta de dados (março de 2012); e a terceira, apenas aqueles que disponibilizavam online os textos completos. Para a recuperação desses textos foram considerados os termos: "Teste de Cloze", "Cloze", "Cloze Test" e "Técnica de Cloze". Nessa terceira etapa foram analisados os seguintes periódicos: Arquivos Brasileiros de Psicologia (publicação quadrimestral), Paidéia (não há informação no site quanto a peridiocidade da publicação), Psicologia: Ciência e Profissão (publicação trimestral), Psicologia Escolar e Educacional (publicação semestral), Psicologia em Estudo - Maringá (publicação quadrimestral), Psicologia: Reflexão e Crítica (publicação quadrimestral), Psic: Revista de Psicologia da Vetor Editora (publicação semestral), Psico-USF (publicação semestral), Psico (publicação trimestral), Psicologia: Teoria e Pesquisa (publicação trimestral), Interação em Psicologia (publicação semestral), Estudos de Psicologia - Campinas (publicação trimestral), Psicologia em Pesquisa (publicação semestral) e Fractal: Revista de Psicologia (publicação semestral).

\section{Fonte}

A amostra do estudo foi composta por 32 artigos recuperados em 14 periódicos que focalizavam o Teste de Cloze entre 2002 e 2012.

\section{Procedimento}

Os artigos foram analisados na íntegra com base em alguns critérios estabelecidos por Witter (1999) e outros considerados relevantes pela autora. Os itens pesquisados foram: (a) a quantidade de artigos publicados por revista e por ano; (b) a distribuição da produção por origem (Sul, Sudeste, Centro-Oeste, Nordeste, Norte, parcerias nacionais e internacionais); (c) a natureza da autoria (individual ou múltipla), bem como o gênero dos autores; (d) a distribuição do tipo de trabalho (relato de pesquisa de campo ou manuscrito teórico); (e) contextos e populações nas quais o instrumento tem sido aplicado; (f) correlação com outros instrumentos (testes, escalas, questionários, entre outros); (g) objetivos dos estudos (diagnóstico - compreensão e inteligibilidade; intervenção e processo de remediação da leitura; validação da técnica); (h) estruturação do texto (Cloze Tradicional, Cloze lexical, Cloze gramatical, Cloze de múltipla escolha e Cloze Cumulativo); (i) parâmetros na omissão sistemática das palavras (retirada de todo quinto, sétimo ou décimo vocábulo; supressão de uma categoria gramatical (adjetivos, substantivos, verbos, entre outras) ou eliminação aleatória de $20 \%$ dos vocábulos do texto); (j) critérios de correção (correção literal ou utilização de sinônimos).

O levantamento de todos os dados foi realizado pela autora da pesquisa e reavaliado por uma pessoa externa ao estudo, separada e concomitantemente, para dar confiabilidade à avaliação. O índice de concordância ficou acima de $90 \%$.

\section{Resultados e discussão}

Considerando-se o objetivo do estudo, num primeiro momento verificou-se a quantidade de artigos publicados por periódico e por ano. A Tabela 1 apresenta os resultados desse levantamento.

Observou-se que das 14 revistas analisadas, nove publicaram apenas um artigo sobre o Teste de Cloze nos últimos dez anos. O maior número de publicações ocorreu nos periódicos 'Psic: Revista de Psicologia da Vetor Editora' ( $n=6 ; 18,8 \%)$, 'Estudos de Psicologia - Campinas' $(n=5$; $15,6 \%$ ) e 'Psicologia: Reflexão e Crítica'( $n=5 ; 15,6 \%)$. Quanto à frequência de publicação por ano, verificou-se um incremento no número de artigos publicados a partir de 2007, sendo os anos de 2007 e 2008 os mais profícuos $\left(n_{2007}=5\right.$; $\left.15,6 \% ; n_{2008}=8 ; 25,0 \%\right)$. O aumento no número de publicações especialmente nos últimos três anos do estudo também foi verificado por outras pesquisas como por exemplo a de Oliveira, Santos e cols. (2007), ao investigarem a produção científica em avaliação psicológica no contexto escolar no período de 1995 a 2004, a de Suehiro, Gaino e cols., (2008) e a de Suehiro e Rueda (2009).

Apesar dos diversos estudos que têm comprovado a versatilidade da técnica e sua utilidade para diferentes objetivos, entre os quais a remediação e o desenvolvimento da compreensão em leitura (Bampi, 2000; Braga, 1981; Carelli, 1992; Castro, 1981; Cunha, 2009; Joly, 1999; Joly \& Lomônaco, 2003; Santos, 2004; entre outros), uma das habilidades mais requisitadas no ensino formal e na vida cotidiana dos indivíduos, considera-se que a técnica ainda tem sido muito pouco explorada no Brasil, o que pode ser comprovado pelo número de artigos ainda restrito no período de dez anos analisado ( $\mathrm{N}=32)$. A baixa produção relativa ao teste é preocupante especialmente porque sua utilização torna-se ainda mais relevante em um contexto no qual diversos dados têm apontado para o fato de que os estudantes, independentemente da etapa de escolaridade na qual se encontrem, compreendem muito pouco do que leem e não têm o hábito de leitura (Carvalho, 2001; Oliveira, 2005; Ribeiro, 2006; Ministério da Cultura, 2011).

Para avaliar a origem da produção, levantou-se a procedência dos artigos. Para tanto, avaliou-se a região, o país e as parcerias regionais e internacionais realizadas. 
Tabela 1. Distribuição geral da quantidade de artigos publicados por revista e por ano (N=32).

\begin{tabular}{lcccccccccccc}
\hline \multicolumn{1}{c}{ Periódicos } & 2002 & 2003 & 2004 & 2005 & 2006 & 2007 & 2008 & 2009 & 2010 & 2011 & Total & $\%$ \\
\hline Arquivos Brasileiros de Psicologia & 0 & 0 & 0 & 0 & 0 & 0 & 0 & 0 & 0 & 1 & 1 & 3,1 \\
Estudos de Psicologia - Campinas & 0 & 0 & 1 & 0 & 1 & 0 & 1 & 1 & 1 & 0 & 5 & 15,6 \\
Fractal: Revista de Psicologia & 0 & 0 & 0 & 0 & 0 & 0 & 0 & 1 & 0 & 0 & 1 & 3,1 \\
Interação em Psicologia & 1 & 1 & 1 & 0 & 0 & 0 & 1 & 0 & 0 & 0 & 4 & 12,5 \\
Paidéia & 0 & 0 & 0 & 0 & 0 & 0 & 1 & 0 & 0 & 0 & 1 & 3,1 \\
Psicologia: Ciência e Profissão & 0 & 0 & 0 & 0 & 0 & 0 & 1 & 0 & 0 & 0 & 1 & 3,1 \\
Psicologia Escolar e Educacional & 0 & 0 & 0 & 0 & 0 & 2 & 1 & 0 & 0 & 0 & 3 & 9,4 \\
Psicologia em Estudo/Maringá & 1 & 0 & 0 & 0 & 0 & 0 & 0 & 0 & 0 & 0 & 1 & 3,1 \\
Psicologia em Pesquisa & 0 & 0 & 0 & 0 & 0 & 1 & 0 & 0 & 0 & 0 & 1 & 3,1 \\
Psicologia: Reflexão e Crítica & 1 & 0 & 0 & 1 & 1 & 0 & 0 & 1 & 1 & 0 & 5 & 15,6 \\
Psicologia: Teoria e Pesquisa & 0 & 0 & 0 & 1 & 0 & 0 & 0 & 0 & 0 & 0 & 1 & 3,1 \\
Psic & 0 & 0 & 0 & 0 & 1 & 2 & 3 & 0 & 0 & 0 & 6 & 18,8 \\
Psico & 0 & 0 & 0 & 0 & 0 & 0 & 0 & 1 & 0 & 0 & 1 & 3,1 \\
Psico-USF & 0 & 0 & 0 & 0 & 0 & 0 & 0 & 0 & 1 & 0 & 1 & 3,1 \\
\hline Total & 3 & 1 & 2 & 2 & 3 & 5 & 8 & 4 & 3 & 1 & & \\
\hline \% & 9,4 & 3,1 & 6,2 & 6,2 & 9,4 & 15,6 & 25 & 12,5 & 9,4 & 3,1 & & 100 \\
\hline
\end{tabular}

Quanto a este aspecto faz-se necessário ressaltar que se consideraram como "parcerias" os textos elaborados entre pesquisadores de diferentes regiões do Brasil e de países distintos. Quando os estudos foram produzidos por pesquisadores de diferentes instituições, porém da mesma região, foram considerados referentes à região da qual os autores eram provenientes.

Os resultados referentes à origem da produção indicaram, por um lado, que a Região Sudeste foi a que mais contribuiu para a divulgação dos conhecimentos produzidos sobre o Cloze ao longo do período analisado, representando a quase totalidade das produções focalizadas $(n=31 ; 96,9 \%)$. Por outro, evidenciaram que as regiões Centro-Oeste, Nordeste e Norte não contribuíram com nenhuma publicação sobre o assunto nos últimos dez anos. A única modificação no cenário em relação a estudos anteriores em produção científica está no fato de que a Região Nordeste começa a figurar nesse novo contexto sob a forma de parceria com outras regiões $(n=1$; $3,1 \%$ ). O fato de o Sudeste figurar como a região de maior produção do conhecimento no país corrobora os resultados obtidos por diversas pesquisas que se detiveram na análise da produção científica, refletindo uma tendência observada na psicologia como um todo (Oliveira e cols., 2007; Souza Filho e cols., 2006, Suehiro \& Rueda, 2009, Suehiro e cols., 2008, no prelo; entre outros).

Quanto ao tipo de autoria (individual ou múltipla), verificou-se que os artigos foram escritos, na maioria, por mais de um autor $(n=29 ; 90,6 \%)$, assim como constatado por Souza Filho e cols. (2006), Oliveira e cols. (2007) e Suehiro e Rueda (2009). A média de autores por artigos foi 2,28, sen- do o mínimo um e o máximo oito. Ao se considerar a amostra total observou-se que a maior parte dos autores foi do sexo feminino ( $n=73 ; 94,8 \%$ ), sendo que na totalidade dos artigos analisados, o primeiro autor também foi do sexo feminino.

Os achados com relação à superioridade feminina na autoria dos trabalhos que têm sido divulgados corroboram a hegemonia verificada em diversos estudos acerca da produção científica no país (Cunha e cols., 2009; Souza Filho e cols., 2006; Suehiro, Cunha, Oliveira e cols., 2007; Suehiro \& Rueda, 2009; Yamamoto e cols., 1999). Ademais, cumpre ressaltar o fato de que, desde a sua criação, a Psicologia têm se constituído como uma profissão tipicamente feminina, o que faz com que essas constatações pareçam algo natural (Bock, 2003; Castro \& Yamamoto, 1998).

No que se refere à análise por tipo de trabalho, buscou-se investigar a modalidade na qual o manuscrito se enquadrava. Para tanto, consideraram-se as categorias relato de pesquisa de campo e manuscrito teórico. Os resultados evidenciaram que todo o material publicado se referia a relatos de pesquisa, confirmando os achados de Souza Filho e cols. (2006), Oliveira e cols. (2007) e Suehiro e Rueda (2009).

Dentre os artigos analisados, 31 referem-se ao contexto escolar, sendo que a maioria focalizou o Ensino Fundamental $(n=15 ; 47 \%)$, vindo a seguir o Ensino Superior $(n=14 ; 43,7 \%)$. Sobre o Ensino Médio foram encontrados artigos $(6,2 \%)$. Do total de artigos, a maioria $(n=16 ; 50,0 \%)$ se propôs avaliar a relação do Teste de Cloze com outros construtos, associando-o a outros testes, escalas, questionários, entre outros. São eles, a Escala de Avaliação de Dificuldades 
na Aprendizagem da Escrita (ADAPE), o WISC III; o Teste de Desempenho Escolar (TDE); o Teste de Reconhecimento de Palavras (EREP); a produção de textos escritos com tema estabelecido; questões sobre as dificuldades percebidas e sobre as estratégias utilizadas para a sua superação; a Escala de Estratégias de Leitura; a Escala de Atitudes de Leitura; a Escala de Avaliação da Escrita (EAVE); a Escala de Estratégias de Aprendizagem e a Escala Beck de Desesperança. Houve ainda o uso de questionários como o de caracterização dos tipos mais frequentes de avaliação utilizados no Ensino Superior; de questões objetivas de matemática e de caracterização das condições de estudo, assim como tarefas de consciência fonológica e de consciência morfológica. Além destes, foram correlacionados com o Cloze as médias acadêmicas ou notas escolares e o desempenho em jogos. Essa tendência em relação ao contexto no qual a técnica tem sido empregada, às etapas de escolarização focalizadas nos estudos, bem como aos testes, escalas, questionários e demais critérios externos correlacionados com o Cloze já havia sido evidenciada por Cunha (2009) e por outros estudos, como os de Bitar (1989), Oliveira, Suehiro e Santos (2004) e Santos e cols. (2006). Os resultados concernentes aos objetivos das produções podem ser visualizados na tabela 2 .
Os dados dispostos na tabela 2 evidenciaram que a maioria das produções envolveu a relação do Cloze - portanto, também da leitura - com outros construtos ( $n=16 ; 50,0 \%)$. Seguiram-se as produções sobre a intervenção e o processo de remediação da leitura $(n=6 ; 18,7 \%)$ e sobre o diagnóstico $(n=5 ; 15,6 \%)$. Do mesmo modo, os achados referentes ao objetivo dos estudos recuperados aqui e os formatos diversificados que a técnica pode assumir, corroboram os de Cunha (2009), tendo em vista que os objetivos de diagnóstico da compreensão em leitura $(n=5 ; 15,6 \%)$ e de programas de intervenção e remediação $(n=6 ; 18,7 \%)$ figuram entre os mais frequentes. Além da predominância daqueles que se basearam no Cloze Tradicional ( $n=28 ; 84,8 \%$ ), com parâmetro de omissão sistemática de todo quinto vocábulo do texto $(n=$ $31 ; 96,8 \%)$ e correção literal $(n=20 ; 63,7)$, ou seja, aquela na qual se considera correta toda palavra exatamente igual à suprimida. Esses resultados corroboram a aplicabilidade do Cloze como técnica de avaliação e remediação da compreensão em leitura, como demonstraram diversos estudos, podendo ser visualizados na tabela 3 (Bampi, 2000; Braga, 1981; Carelli, 1992; Castro, 1981; Coelho \& Correa, 2010; Hussein, 2008; Joly, 1999; Joly \& Lomônaco, 2003; Oliveira, Boruchovitch e cols., 2007; Santos \& Oliveira, 2010; Oliveira \& Santos, 2008; Santos, 2004; Zucoloto \& Sisto, 2002).

Tabela 2. Distribuição das produções em função dos seus objetivos $(\mathrm{N}=32)$.

\begin{tabular}{lcc}
\hline \multicolumn{1}{c}{ Objetivo } & $F$ & $\%$ \\
\hline Diagnóstico, compreensão e inteligibilidade & 5 & 15,6 \\
Intervenção e processo de remediação da leitura & 6 & 18,7 \\
Relação com outros construtos & 16 & 50,0 \\
Validação da técnica & 4 & 12,5 \\
Produção científica & 1 & 3,1 \\
\hline Total & 32 & 100 \\
\hline
\end{tabular}

Tabela 3 - Distribuição da produção por estruturação do texto e correção empregados (N=31).

\begin{tabular}{lrrlrr}
\hline \multicolumn{1}{c}{ Estruturação do Texto } & \multicolumn{1}{c}{ F } & \multicolumn{1}{c}{$\%$} & Correção & $F$ & $\%$ \\
\hline Tradicional & 28 & 84,8 & Literal & 21 & 63,7 \\
Lexical & 0 & 0 & Sinônima & 1 & 3,0 \\
Gramatical & 0 & 0 & Não há relato & 10 & 30,3 \\
Múltipla escolha & 3 & 9,2 & Não se aplica & 1 & 3,0 \\
Cumulativo & 1 & 3,0 & & & \\
Não se aplica & 1 & 3,0 & & & \\
\hline Total & 33 & 100 & & 33 & 100 \\
\hline
\end{tabular}




\section{À guisa de conclusão}

O objetivo do presente estudo foi realizar um levantamento dos trabalhos publicados sobre o Teste de Cloze no período de 2002 a 2012, em duas bases de dados online. Isso porque se considerou que o acesso a produções em bases de dados desse tipo tem permitido uma disseminação muito mais rápida do conhecimento produzido nas diversas áreas do conhecimento.

O número de estudos aqui recuperado pode ser considerado ainda pequeno, especialmente ao se considerar a importância da compreensão em leitura para qualquer cidadão. Ao lado disso, é preciso ressaltar que apesar de o Cloze ser um instrumento de fácil aplicação e não ser restrito aos psicólogos, no que se refere especificamente à Psicologia, muito ainda há para se explorar, especialmente no que concerne à sua cientificidade. Outro dado que chama a atenção é o fato de que o Ensino Médio tem sido muito pouco pesquisado, o que, em consonância com as atuais avaliações do governo brasileiro, ressalta a importância de estudos com essa população.

As categorias de análise focalizadas neste estudo foram: quantidade de artigos publicados por revista e por ano; distribuição da produção por origem; natureza da autoria e gênero dos autores; distribuição do tipo de trabalho; contextos e populações nas quais o instrumento tem sido aplicado; correlação com outros instrumentos; objetivos dos estudos; estruturação do texto e, por fim, parâmetros na omissão de palavras e critérios de correção. Embora se tenham empregado categorias amplamente difundidas para estudos desse porte e outras relevantes para o estudo da técnica, considera-se que devem ser realizadas pesquisas mais aprofundadas sobre o objetivo dos estudos, mais especificamente sobre suas evidências de validade e as análises realizadas, e ainda sobre os resultados obtidos. Neste sentido, em face das limitações desta pesquisa - como o foco em apenas duas bases de dados online e a quantidade ainda restrita de estudos voltados para a análise da produção científica que possibilitem maior respaldo para seus achados - considera-se que estudos com um maior aprofundamento devem ser realizados levando em consideração as possibilidades de investigação citadas anteriormente. Pesquisas desse porte são relevantes não apenas por possibilitarem o desenho e o desenvolvimento da área, mas, sobretudo, por funcionarem como um indicativo das lacunas que precisam ser preenchidas, contribuindo assim para a disseminação, a qualidade e o progresso do conhecimento produzido.

\section{Referências}

Aguiar Netto, M. C. (1988). A produção do conhecimento psicológico fora do espaço acadêmico. Em Conselho Federal de Psicologia (Org.), Quem é o psicólogo brasileiro (pp.123-137). São Paulo: Edicon.

Arouca, E. A. (1997). Universitário, redação e SPI. Psicologia Escolar e Educacional, 1(2), 51-58

Bampi, M. L. F. (2000). Programa para o desenvolvimento da leitura e escrita: faz de conta e criatividade. Tese de Doutorado, Universidade de São Paulo, São Paulo-SP.

Bensoussan, M. (1990). Redundancy and the cohesion Cloze. Journal of Research in Reading, 13(1), 18-37.

Bitar, M. L. (1989). Eficiência dos instrumentos de avaliação em leitura. Dissertação de Mestrado, Pontifícia Universidade Católica de São Paulo, São Paulo-SP.

Bock, A. (2003). Quarenta e um anos de profissão no Brasil. Disponível em http:// www.pol.org.br/publicacoes/materia. Acessado em 03/05/2007.

Bormuth, J. R. (1968). Cloze Test readability: Criterion references scores. Journal of Educational Measurement, 5, 189-196.

Braga, S. M. L. (1981). Remediação da leitura: um estudo com escolares de primeiro grau utilizando a técnica de cloze. Dissertação de Mestrado, Universidade de São Paulo, São Paulo-SP.

Braga, S. M. L. (1986). Produção de texto e o modelo operante: treino de repertórios específicos. Tese de Doutorado, Universidade de São Paulo, São Paulo-SP.

Capovilla, S. L., \& Santos, A. A. A. (2001). Avaliação da influência de atividades extramuros no desenvolvimento pessoal de universitários. Psico-USF, 6(2), 49-58.

Carelli, A. E. (1992). Teste da eficiência de programas de compreensão e leitura crítica. Dissertação de Mestrado, Pontifícia Universidade Católica de Campinas, Campinas-SP.

Carvalho, M. P. (2001). Estatísticas de desempenho escolar: o lado avesso. Educação \& Sociedade, 77, 231-252.

Castelo Branco, S. M. C. (1992). Estudo do desenvolvimento de leitura oral em escolares de $1^{a}$ a $4^{a}$ série do primeiro grau. Dissertação de Mestrado, Pontifícia Universidade Católica de São Paulo, São Paulo-SP.

Castro, A. E. F., \& Yamamoto, O. Y. (1998). A psicologia como profissão feminina: apontamentos para estudo. Estudos de Psicologia, 3(1), 147-158.

Castro, M. L. U. (1981). Programa de leitura recreativa: efeito de dois procedimentos de treino com escolares de quarta série do $1^{\circ} \mathrm{grau}$. Dissertação de Mestrado, Universidade de São Paulo, São PauloSP.

Coelho, C. L. G., \& Correa, J. (2010). Desenvolvimento da compreensão leitora através do monitoramento da leitura. Psicologia: Reflexão e Crítica, 23(3), 575-581. 
Cohen, J. H. (1975). The effect of content area material on Cloze test performance. Journal of Reading, 19(3), 247-250.

Cunha, N. B. (2009). Pesquisas com o teste de Cloze no Brasil. Em A. A. A. dos Santos, E. Boruchovitch \& K. L. de Oliveira (Orgs.), Cloze: Um instrumento de diagnóstico e intervenção (pp. 80-117). São Paulo: Casa do Psicólogo.

Cunha, N. B., Suehiro, A. C. B., Oliveira, E. Z., Pacanaro, S. V., \& Santos, A. A. A. (2009). Produção Científica da Avaliação da leitura no Contexto Escolar. Psico, 40(1), 17-23.

Freitas, M. H. A. (1998). Avaliação da produção científica: considerações sobre alguns critérios. Psicologia Escolar e Educacional, 2(3), 211-228.

Garrido, E. (1979). A técnica cloze e a compreensão da leitura. Dissertação de Mestrado, Universidade Estadual de Campinas, Campinas-SP.

Garrido, E. (1988). O ensino da Filosofia no $2^{\circ}$ grau e a compreensão de textos: um levantamento em São Paulo e uma aplicação da técnica de Cloze. Tese de Doutorado, Universidade de São Paulo, São Paulo-SP.

Giordano, G. (1985). Learning to reading erased text. Academic Therapy, 20(8), 317-322.

Grant, P. L. (1979). The cloze procedure as an instuctional device. Journal of Reading, 28(4), 705-719.

Haugh, O. M. (1975). Reading and readability. Em Annual Meeting of the National Council of Teachers of English (pp. 1-11), 65, San Diego.

Helfeldt, J. P., \& Henk, W. A. (1985). Unfulness of conventional vs total random cued cloze tests as measure of reading comprehension. Journal of Reading, 28(8), 719-725.

Hines, T. C., \& Warren, J. (1978). A computadorized technique for producing cloze test material. Educational Technology, 18(9), 5658.

Hussein, C. L. (2008). Avaliação de treino de leitura compreensiva e crítica: estudo com universitários. Psicologia Escolar e Educacional, 12(2), 401-411.

Joly, M. C. R. A. (1999). Microcomputador e criatividade em leitura e escrita no ensino fundamental. Tese de Doutorado, Universidade de São Paulo, São Paulo-SP.

Joly, M. C. R. A., \& Lomônaco, J. F. B. (2003). Avaliando a compreensão de leitura no ensino fundamental: uma comparação entre o instrumento eletrônico e o impresso. Boletim de Psicologia, 53(119), 131-147.

Kletzien, S. B. (1991). Strategy use by good and poor comprehenders reading expository text differing levels. Reading Research Quaterly - International Reading Association, XXVI(1), 67-86.

Kopke Filho, H. (2001). Estratégias em compreensão da leitura: conhecimento e uso por professores de língua portuguesa. Tese de Doutorado, Universidade de São Paulo, São Paulo-SP.

Ministério da Cultura (2011). Lendo o Brasil. Recuperado: 08 mar 2012. Disponível: http://www.cultura.gov.br/site/2011/02/08/lendoo-brasil-artigo.

Neves, T. R. P. (1997). O processamento da informação na leitura de textos em sala de aula. Dissertação de Mestrado, Universidade Federal de Santa Maria, Santa Maria-RS.

Oliveira, J. B. A. (2005). ABC do alfabetizador (3a ed.). Belo Horizonte: Alfa Educativa.

Oliveira, K. L., Boruchovitch, E., \& Santos, A. A. A. (2007). Compreensão de leitura em alunos de sétima e oitava séries do ensino fundamental. Psicologia Escolar e Educacional, 11(1), 4149.

Oliveira, K. L., Boruchovitch, E., \& Santos, A. A. A. (2009). A técnica de Cloze na avaliação da compreensão em leitura. Em A. A. A. dos Santos, E. Boruchovitch \& K. L. de Oliveira (Orgs.), Cloze: Um instrumento de diagnóstico e intervenção (pp. 46-77). São Paulo: Casa do Psicólogo.

Oliveira, K. L., Cantalice, L. M., Joly, M. C. A., \& Santos, A. A. A. (2006). Produção científica de 10 anos da revista Psicologia Escolar e Educacional (1996/2005). Psicologia Escolar e Educacional, 10(1), 283-292.

Oliveira, K. L., \& Santos, A. A. A. (2008). Estudo de intervenção para a compreensão em leitura na universidade. Interação em Psicologia, 12(2), 169-177.

Oliveira, K. L., Santos, A. A. A., Noronha, A. P. P., Boruchovitch, E., Cunha, C. A., Bardagi, M. P., \& Domingues, S. F. S. (2007) Produção científica em avaliação psicológica no contexto escolar. Psicologia Escolar e Educacional, 11(1), 239-251.

Oliveira, K. L., Suehiro, A. C. B., \& Santos, A. A. A. (2004). Avaliação da Aprendizagem no Ensino Superior: Estudo da Relação com Compreensão em Leitura Em: C. Machado, L. S. Almeida, M. Gonçalves \& V. Ramalho (Orgs.), Avaliação Psicológica: Formas e Contextos (pp. 216-223). Ed. Braga: Psiquilíbrios.

Page, W. D. (1975). The post-oral reading Cloze test: New link between oral reading and comprehension. Journal of Reading Behavior, 7(4), 383-389.

Pellegrini, M. C. K. (1996). Avaliação dos níveis de compreensão e atitudes frente à leitura em universitários. Dissertação de Mestrado, Universidade São Francisco, Bragança Paulista-SP. 
Pinto, C. J. A., Alvarenga, M. A. P., \& Kock, R. A. A. (1997). Hábitos de leitura e compreensão de texto entre universitários. Em G. P. Witer (Org.), Leitura e universidade (pp. 133-165). Campinas: Alínea.

Porter, D. (1979). Cloze procedure and quivalence. Language Learning, 28(2), 333-341.

Rankin, E. F. (1970). Grade level interpretation of cloze readability scores. National Reading Conference ${ }_{\perp}$ Saint Petersburg.

Ribeiro, V. M. (2006). Analfabetismo e alfabetismo funcional no Brasil. Recuperado: $20 \mathrm{dez}$ 2006. Disponível: http//www. reescrevendoaeducacao.com. br/pages. . php? recid $=28$

Riley, J. D. Progressive close as a remedial technique. The reading teacher, 39(6), 576-581.

Rinaudo, M. C. \& Olmos, G. V. (1996). Aprendizaje cooperativo y comprensión de la lectura: una experiencia con estudiantes universitarios. Lectura y Vida, 1(17), 25-38.

Sampaio, I. S., \& Santos, A. A. A. (2002). Leitura e redação entre universitários: avaliação de um programa de intervenção. Psicologia em Estudo, 7(1), 31-38.

Santos, A.A.A. (1990). Compreensão em leitura na universidade: um estudo comparativo entre dois procedimentos de treino. Estudos de Psicologia, 7(2), 39-53.

Santos, A.A.A. (1991). Desempenho em leitura: um estudo diagnóstico da compreensão e hábitos de leitura entre universitários. Estudos de Psicologia, 8(1), 6-19.

Santos, A.A.A. (1994). Programas de remediação: uma alternativa para o desenvolvimento da compreensão em leitores adultos. ProPosições, 5(1/13), 115-122.

Santos, A.A.A. (1997). Psicopedagogia no $3^{\circ}$ grau: avaliação de um programa de remediação em leitura e estudo. Pro-Posições, 8(1), 27-37.

Santos, A.A.A. (2004). O Cloze como técnica de diagnóstico e remediação da compreensão em leitura. Interação em Psicologia, 8(2), 217-226.

Santos, A. A. A. (2005). O Teste de Cloze como instrumento de diagnóstico e de desenvolvimento da compreensão em leitura. Relatório Técnico de Bolsista Produtividade enviado ao CNPq, Universidade São Francisco, Itatiba-SP.

Santos, A. A. A., \& Oliveira, E. Z. (2010). Avaliação e desenvolvimento da compreensão em leitura no ensino fundamental. Psico-USF, 15 (1), 81-91.

Santos, A. A. A., Suehiro, A. C. B., \& Oliveira, K. L. (2004). Habilidades em compreensão da leitura: um estudo com alunos de psicologia Estudos de Psicologia, 21(2), 29-41.
Santos, A. A. A., Vendramini, C. M. M., Suehiro, A. C. B., \& Santos, L. A. D. (2006). Leitura compreensiva e utilização de estratégias de aprendizagem em alunos de Psicologia. Estudos de Psicologia, 23(1), 83-91.

Souza Filho, M. L., Belo, R., \& Gouveia, V. V. (2006). Testes psicológicos: análise da produção científica brasileira no período de 2000-2004. Psicologia: Ciência e Profissão, 26(3), 478-489.

Suehiro, A. C. B. \& Rueda, F. J. M. (2009). Avaliação Psicológica: um estudo da produção científica de 2002 a 2007. Avaliação Psicológica, 8(1), 131-139.

Suehiro, A. C. B., \& Santos, A. A. A. (2009). O Teste de Cloze e o Desenvolvimento Percepto-Motor no Início da Escolarização. Em A. A. A. dos Santos, E. Boruchovitch \& K. L. de Oliveira (Orgs.), Cloze: Um instrumento de diagnóstico e intervenção (pp. 188225). São Paulo: Casa do Psicólogo.

Suehiro, A. C. B., Cunha, N. B., \& Santos, A. A. A. (2007). Avaliação da escrita no contexto escolar entre 1996 e 2005. Psic, 8(1), 6170.

Suehiro, A. C. B., Cunha, N. B., Oliveira, E. Z., \& Pacanaro, S. V. (2007). Produção Científica da Revista Psico-USF de 1996 a 2006. Psico-USF, 12(1), 327-334.

Suehiro, A. C. B., Gaino, S. B. \& Meireles, E. (2008). Produção científica sobre o Teste Gestáltico Viso-Motor de Bender entre 1999 e 2008. Psic, 9(2), 173-181.

Suehiro, A. C. B., Gaino, S. B., \& Meireles, E. (no prelo). Teste Gestáltico Viso-Motor de Bender: levantamento das pesquisas sobre parâmetros psicométricos entre 2002 e 2011. Psico.

Suehiro, A. C. B., Rueda, F. J. M., Oliveira, E. Z., \& Pacanaro, S. V. (2009). Avaliação do Autoconceito no Contexto Escolar em Periódicos Brasileiros. Psicologia: Ciência e Profissão, 29(1), 1829

Taylor, W. L. (1953). Cloze procedure: a new tool for measuring readabilit. Journalism Quarterly, 30, 415-433.

Vicentelli, H. (1999). Problemática de la lectura en estudiantes universitarios. Psicologia Escolar e Educacional, 3(3), 195-202.

Witter, C. (1996). Psicologia Escolar: produção científica, formação e atuação (1990-1994). Tese de doutorado, Universidade de São Paulo, São Paulo.

Witter, G. P. (1996). Avaliação da produção científica sobre leitura na universidade. Psicologia Escolar e Educacional, 1(1), 31-37.

Witter, G. P. (1999). Metaciência e leitura. Em G. P. Witter (Org.), Leitura: textos e pesquisas (pp. 13-22). Campinas, SP: Alínea. 
Witter, G. P. (2006). Produção científica: escalas de avaliação. Em D. A. Poblacion, G. P. Witter \& J. F. M. da Silva (Orgs.), Comunicação e produção científica: contexto, indicadores, avaliação (pp. 287311). São Paulo: Angellara.

Yamamoto, O. H, Souza, C. C., \& Yamamoto, M. E. (1999). A produção científica na psicologia: uma análise dos periódicos brasileiros no período 1990-1997. Psicologia: Reflexão e Crítica, 12(2), 549-565.
Zucoloto, K. A. (2001). A compreensão da leitura em crianças com dificuldade de aprendizagem na escrita. Dissertação de Mestrado, Universidade Estadual de Campinas, Campinas-SP.

Zucoloto, K. A., \& Sisto, F. F. (2002). Dificuldades de aprendizagem em escrita e compreensão em leitura. Interação, 6(2), 157-166.

Recebido em: 03-04-2012

Reformulado em : 01-08-2012

25-10-2012

Aprovado em:17-11-2012

\section{Sobre a autora}

Adriana Cristina Boulhoça Suehiro (dricbs@yahoo.com.br)

Psicóloga. Doutora em Psicologia pelo Programa de Pós-Graduação Stricto Sensu em Psicologia da Universidade São Francisco e docente da Universidade Federal do Recôncavo da Bahia. Líder e pesquisadora do Laboratório de Instrumentação e Avaliação Psicológica da Universidade Federal do Recôncavo da Bahia (LABIAP).

Endereço: Avenida Carlos Amaral, 1015. Cajueiro, Santo Antônio de Jesus - BA. CEP: 44574-490 